\title{
Remifentanil y propofol con infusión controlada por objetivo para legrado uterino
}

\author{
Alegre Andrade P. ${ }^{1}$, Mamani Ortiz Y. ${ }^{2}$ \\ 1 Hospital Obrero N² "Caja Nacional de Salud", Cochabamba, Bolivia. \\ 2 Instituto de Investigaciones Biomédicas, UMSS, Cochabamba, Bolivia.
}

Objetivo: Analizar el comportamiento hemodinámico frente al manejo anestésico con remifentanilo y propofol con infusión controlada por objetivo en pacientes sometidas a legrado uterino por aborto incompleto.

Métodos: Se realizó un ensayo clínico no controlado utilizando un protocolo de atención aprobado por el servicio de anestesiología obstétrica del Hospital Obrero №2, entre enero a mayo de 2019, ingresado 39 pacientes sometidas a legrado uterino instrumental; usando el modelo Minto para remifentanil y el modelo Schnider para Propofol para la infusión controlada. La valoración de las diferencias estadísticas en los parámetros hemodinámicos se realizó mediante comparación de medias por $\mathrm{t}$ de student.

Resultados: La edad media del estudio es $34,77 \pm 7,9$ años, tiempo quirúrgico de $12 \pm 2,9$ minutos. El tiempo anestésico de $15 \pm 3$ minutos; el tiempo del alta de la recuperación anestésica a salas de $14 \pm 3,3$ minutos. La dosis de inducción del remifentanilo utilizada fue de 4,8 $\pm 0,3 \mathrm{ng} / \mathrm{ml}(\mathrm{p}>0,001)$ y la dosis de inducción del propofol fue de 3,77 $\pm 0,4 \mathrm{mcg} / \mathrm{ml}(\mathrm{p}>0,001)$. Las dosis de mantenimiento del remifentanilo de 4,56 $\pm 0,5 \mathrm{ng} / \mathrm{ml}$ y la dosis del propofol es de $3,8 \pm 0,6 \mathrm{mcg} / \mathrm{ml}$ ( $\mathrm{p}>0,001)$. No se encontró complicaciones ni hallazgos secundarios por el uso de los medicamentos.

Conclusiones: El uso del sistema de infusión controlado por objetivo (TCI) para remifentanilo en el modelo de Minto y modelo de Schinder para el propofol proporciona a las pacientes buena estabilidad hemodinámica

https://doi.org/10.25237/congresoclasa2019.18 